\title{
Posterior Cerebellar Volume and Executive Function in Young Adults With Congenital Heart Disease
}

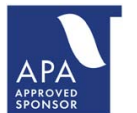

NS is approved by the American Psychological Association to sponsor Continuing Education for psychologists. INS maintains responsibility for this program and its content.

\author{
Eric S. Semmel, ${ }^{1}$ Vonetta M. Dotson, ${ }^{1}$ Thomas G. Burns, ${ }^{2}$ William T. Mahle, ${ }^{2,3}$ And Tricia Z. King ${ }^{1}$ \\ ${ }^{1}$ Georgia State University, Department of Psychology and Neuroscience Institute, Atlanta, Georgia \\ ${ }^{2}$ Children's Healthcare of Atlanta, Atlanta, Georgia \\ ${ }^{3}$ Emory University School of Medicine, Atlanta, Georgia
}

(Received November 15, 2017; Final Revision February 9, 2018; Accepted March 29, 2018; First Published OnLine May 30, 2018)

\begin{abstract}
Objectives: As the number of adolescents and young adults (AYAs) surviving congenital heart disease (CHD) grows, studies of long-term outcomes are needed. CHD research documents poor executive function (EF) and cerebellum (CB) abnormalities in children. We examined whether AYAs with CHD exhibit reduced EF and CB volumes. We hypothesized a double dissociation such that the posterior $\mathrm{CB}$ is related to $\mathrm{EF}$ while the anterior $\mathrm{CB}$ is related to motor function. We also investigated whether the $\mathrm{CB}$ contributes to $\mathrm{EF}$ above and beyond processing speed. Methods: Twenty-two AYAs with CHD and 22 matched healthy controls underwent magnetic resonance imaging and assessment of EF, processing speed, and motor function. Volumetric data were calculated using a cerebellar atlas (SUIT) developed for SPM. Group differences were compared with $t$ tests, relationships were tested with Pearson's correlations and Fisher's $\mathrm{r}$ to $\mathrm{z}$ transformation, and hierarchical regression was used to test the CB's unique contributions to EF. Results: CHD patients had reduced CB total, lobular, and white matter volume $(d=.52-.99)$ and poorer EF $(d=.79-1.01)$ compared to controls. Significant correlations between the posterior $\mathrm{CB}$ and $\mathrm{EF}(r=.29-.48)$ were identified but there were no relationships between the anterior $\mathrm{CB}$ and motor function nor EF. The posterior $\mathrm{CB}$ predicted $\mathrm{EF}$ above and beyond processing speed $(p \mathrm{~s}<.001)$. Conclusions: This study identified a relationship between the posterior $\mathrm{CB}$ and $\mathrm{EF}$, which appears to be particularly important for inhibitory processes and abstract reasoning. The unique $\mathrm{CB}$ contribution to EF above and beyond processing speed alone warrants further study. (JINS, 2018, 24, 939-948)
\end{abstract}

Keywords: Heart defects, Congenital, Cerebellum, Magnetic resonance imaging, Inhibition, Hypoxia, Motor skills

\section{INTRODUCTION}

Congenital heart disease (CHD) is the most common birth defect in the United States, occurring in $1 \%$ of births each year (Hoffman, Kaplan, \& Liberthson, 2004). Even though CHD remains the leading cause of infant death due to birth defect, advancements in cardiology have led to rapidly falling mortality rates, decreasing by $24.1 \%$ between 1999 and 2006 (Gilboa, Salemi, Nembhard, Fixler, \& Correa, 2010; Yang et al., 2006). There are now more than 1,000,000 adults with $\mathrm{CHD}$ in the United States, a number which is expected to grow by $5 \%$ each year (Jackson, Misiti, Bridge, Daniels, \& Vannatta, 2015; Marelli, Mackie, Ionescu-Ittu, Rahme, \& Pilote, 2007). This necessitates the further study and understanding of longterm outcomes in CHD, a topic which until recently has been relatively neglected in the literature.

Correspondence and reprint requests to: Tricia Z. King, Department of Psychology, Georgia State University, P.O. Box 5010, Atlanta, GA 303025010. E-mail: tzking@gsu.edu
As the CHD population ages, areas of concern for these patients include academic, occupational, and behavioral concerns as well as health-related quality of life (Geyer, Norozi, Buchhorn, \& Wessel, 2009; van Rijen et al., 2003). Important to all of these are cognitive outcomes, especially executive function (EF). CHD patients are at risk for compromised $\mathrm{EF}$, which can impact work and school performance and cause difficulties for young adults who are transitioning to manage their own medical care (Murdaugh, King, \& O'Toole, 2017).

\section{COGNITIVE OUTCOMES AND BRAIN DEVELOPMENT IN CHD}

EF deficits have been consistently found in children with CHD, with some studies determining the rate of impairment is double that in healthy controls (Bellinger et al., 2011; Bergemann et al., 2015; Cassidy, White, DeMaso, Newburger, \& Bellinger, 2015; Gaynor et al., 2010; Sanz et al., 2017). Some research has shown these deficits lasting 
into adolescence and adulthood, confirming the need for more research on older CHD patients (Daliento et al., 2005; Ilardi, Ono, McCartney, Book, \& Stringer, 2017; Klouda, Franklin, Saraf, Parekh, \& Schwartz, 2017; von Rhein et al., 2014). A recent review found only one study of adults with CHD that measured EF (Daliento et al., 2005; Tyagi et al., 2014). They found that patients performed worse on several EF tests, particularly on the Tower.

Since this review, there have been few new studies of EF in adults with CHD (Ilardi et al., 2017; King et al., 2016; Klouda et al., 2017). Our group found deficits in working memory and informant report of EF (King et al., 2016). Klouda and colleagues found no differences between moderate CHD and controls, but individuals with severe CHD exhibited various deficits, including EF. Finally, Ilardi and colleagues found that adults with CHD performed significantly more poorly on measures of visuospatial construction and working memory and that individuals at higher risk for neurological disruption performed more poorly on the D-KEFS Category Switching subtest.

In addition to cognitive impairment, studies commonly find disrupted neurodevelopment, cerebral immaturity and reductions in volume, as well as pre- and post-operative white matter injury (Beca et al., 2013; Mebius, Kooi, Bilardo, \& Bos, 2017; Peyvandi et al., 2016). These abnormalities result from subtle genetic differences and copy number variations (the number of times a given gene is coded in an individual's DNA) in addition to hypoxia and suboptimal blood flow to the developing brain in utero (Licht et al., 2004; Mahle et al., 2000; Sun et al., 2015). Some lifesaving surgeries require the use of cardiopulmonary bypass, deep hypothermic circulatory arrest, or regional cerebral perfusion, which can add to injury (du Plessis, 1999; Wray, 2001). Mahle et al. (2002) and Beca et al. (2013) found new white matter injury in neonates after surgery and determined that these injuries are associated with the amount of time spent on bypass, lactate levels, brain maturity, and pre-operative injury.

One area of the brain that is particularly vulnerable to hypoxic injury is the cerebellum (CB), which has been documented as reduced in volume in the $\mathrm{CHD}$ population (Owen et al., 2014; von Rhein et al., 2014; Zeng et al., 2015). In typically developing fetuses, the $\mathrm{CB}$ develops rapidly between 24 and 40 weeks gestation, increasing in volume five-fold (Volpe, 2009). Consequently, it has been proposed that the $\mathrm{CB}$ is particularly vulnerable due to the high metabolic demands of such rapid growth (Limperopoulos et al., 2005; Owen et al., 2014). Reduced CB volume has been found in both fetuses and infants with CHD (Ortinau et al., 2012; Owen et al., 2014; Zeng et al., 2015). Studies of premature infants, who are also vulnerable to hypoxia, contribute more findings of abnormal CB development. Allin et al. (2001) found significantly reduced CB volumes in adolescents who were born prematurely, suggesting that developmental abnormalities in $\mathrm{CB}$ structure can persist long-term in the premature population. Furthermore, these differences persisted even when controlling for whole brain volume, sex, and socioeconomic status. This robust finding strongly supports the proposition that $\mathrm{CB}$ structure is particularly sensitive to developmental disruption.

\section{CEREBELLUM AND EF}

The cortico-centric view of cognition has traditionally been the dominant framework for conceptualizing higher-order processes in neuropsychology. However, many researchers argue that focusing exclusively on cortical bases of higherorder cognition neglects to recognize the important roles subcortical structures like the CB, basal ganglia, and hippocampus. Some research has proposed that the CB facilitates "cognitive efficiency" through the formation of internal models, in which the CB learns through repetition and stores the most efficient schematic for a given cognitive process (Ailion et al., 2016; Koziol et al., 2014). In this way, the CB refines cognitive processes similarly to how it is believed to automate movement. Previously, processing speed has been used as a proxy measurement for the cognitive efficiency the $\mathrm{CB}$ is hypothesized to facilitate (Ailion et al., 2016).

Neuroanatomically, the CB shares multiple bidirectional connections with prefrontal regions and previous findings specifically implicate the posterior lobe of the $\mathrm{CB}$ in cognitive processes, with anterior areas associated with motor functions (Koziol, Budding, \& Chidekel, 2010; Stoodley \& Schmahmann, 2010). There is a growing literature associating CB structure with poorer EF performance in clinical samples, including in children born preterm, attention-deficit/hyperactivity disorder, and spina bifida myelomeningocele (SBM) (Allin et al., 2001; Bolduc et al., 2012; Dennis \& Barnes, 2010; Juranek, Dennis, Cirino, El-Messidi, \& Fletcher, 2010; Koziol, Budding, \& Chidekel, 2012; Limperopoulos et al., 2007; Mahone, Zabel, Levey, Verda, \& Kinsman, 2002; Martin \& Kitzman, 2017; Stoodley \& Schmahmann, 2010). The SBM population is particularly relevant due to the ubiquity of Chiari-II malformations in this group, which results in posterior fossa and $\mathrm{CB}$ abnormalities as well as challenges with attention and EF (Juranek et al., 2010).

Furthermore, imaging studies have found CB activation during Tower of London performance with functional magnetic resonance imaging (fMRI) and positron emission tomography methods (Schall et al., 2003) as well as poorer auditory attention in a CHD sample with reduced white matter integrity in the CB (Brewster, King, Burns, Drossner, $\&$ Mahle, 2015; Schall et al., 2003). Few other studies have examined the relationship between the $\mathrm{CB}$ and EF abilities, therefore, this study may help to extend the literature on EF-CB relationships and its role in outcomes in those with CHD.

To date, no studies have evaluated the relationship between $\mathrm{CB}$ volume and EF in adults with CHD. Therefore, this study used structural MRI analysis and neuropsychological assessment in a sample of AYAs with CHD and healthy controls. First, we expected to find reduced total and lobular CB volumes as well as lower performance on measures of EF, processing speed, and motor function in the CHD sample. Second, we computed correlations between $\mathrm{CB}$ volume, EF performance, and motor functioning, expecting a double dissociation such 
that the posterior $\mathrm{CB}$ would be related to $\mathrm{EF}$ outcomes while the anterior $\mathrm{CB}$ would be associated with motor function. Third, we conducted hierarchical regression to determine whether the posterior $\mathrm{CB}$ contributes to $\mathrm{EF}$ above and beyond processing speed. Previous research has characterized the CB as facilitating speeded cognition but finding relationships between the $\mathrm{CB}$ and $\mathrm{EF}$ even while controlling for processing speed would suggest that it plays a role in facilitating efficient EF in a more nuanced way. Finally, we conducted an a priori exploratory analysis to explore differences in our outcome variables by diagnosis severity group, expecting that more severe diagnoses would exhibit lower outcomes.

\section{METHODS}

\section{Procedures}

All procedures were approved by the IRB and completed in accordance with the Helsinki Declaration. Participants were identified from the pediatric cardiology database at Children's Healthcare of Atlanta and Emory University. The study population was comprised of participants with CHD diagnoses that required surgery with cardiopulmonary bypass in infancy. Diagnoses included both biventricular anatomy, including D-transposition of the great arteries and total anomalous pulmonary venous connection; and single ventricle anatomy, including a double inlet left ventricle, hypoplastic left heart syndrome, tricuspid atresia, and pulmonary atresia with a hypoplastic right ventricle. Participants were eligible if they were between the ages of 16 and 22 and were able to read English and complete questionnaires.

Participants were excluded due to severe and persistent mental or physical disability (e.g., cerebral palsy, genetic syndromes), prematurity (less than 34 weeks gestational age), history of cerebral vascular events, history of brain tumor or traumatic brain injury, visual or hearing impairment, ferromagnetic implants/devices, or residence more than 60 miles from downtown Atlanta. The exclusion of individuals with severe disabilities and cerebral vascular events was due to our desire to study the impact of CHD itself on neuroanatomical and cognitive outcomes and reduce confounding factors posed by other conditions. Given that the rate of stroke in this population is fairly low, with one report quantifying it at $3 \%$, the exclusion of cerebral vascular events should not impact the representativeness of the sample (Barker et al., 2005). Also, while some studies define prematurity at a later gestational age (e.g., 36 weeks), $60 \%$ of children with CHD meet criteria for prematurity with this criterion (Williams et al., 2010). Therefore, to avoid excluding too many representative CHD subjects, we defined prematurity at 34 weeks gestational age.

Recruitment consisted of a telephone interview with eligible AYAs (or family members authorized to give consent for individuals under the age of 18). Forty-five individuals met the inclusion criteria and were contacted by phone. Twenty-six participants agreed to enroll in the study (58\%), while 19 did not participate due to lack of interest, scheduling conflicts, or missing their appointment. There were no significant differences in the proportion of single ventricle diagnoses or age at first surgery between those who enrolled and declined to participate. This suggests that our sample represents the distribution of diagnosis severity in the CHD population. Twenty-three participants completed both neuroimaging and neuropsychological assessment. Eight controls were recruited for this study, and another 15 were selected from a larger study of individuals from the Atlanta community and were recruited from university research pools, community flyers, and word of mouth from clinical participants (King, Na, \& Mao, 2015). Controls were matched as closely as possible by age, sex, and race. One CHD participant and one control participant had unusable imaging data due to motion artifact, therefore, the final sample was comprised of 22 participants in each group. Forty-eight percent of our CHD group carried single ventricle diagnoses (more severe diagnoses with one functioning ventricle) while the remaining $52 \%$ had biventricular diagnoses (Table 1).

\section{Imaging Parameters}

Participants were scanned in a 3 Tesla Siemens Trio MRI scanner. Anatomical scans were high-resolution T1-weighted

Table 1. Demographic and Medical Variables

\begin{tabular}{|c|c|c|c|c|}
\hline Variable & $\mathrm{CHD}$ & Control & $t / \chi^{2}$ & $d$ \\
\hline Subgroup size & 22 & 22 & - & \\
\hline $\operatorname{Sex}(\%$ male $)$ & 69.6 & 69.6 & 0.00 & \\
\hline Race (\% white) & 73.9 & 60.9 & 1.22 & \\
\hline Age at examination $(S D)$ & $18.05(1.62)$ & $18.53(1.69)$ & 0.97 & 0.29 \\
\hline Handedness (\% right) & 86.4 & 72.7 & 1.26 & \\
\hline Mean years education $(S D)$ & $11.7(1.42)$ & $12.7(1.15)$ & 1.99 & 0.60 \\
\hline Diagnosis groups (\% SV/BV) & $48 / 52$ & - & - & - \\
\hline No. of surgeries (SV/BV) & 2.73 (range $1-4) / 1.09$ (range $1-2$ ) & - & - & - \\
\hline Age in days at first surgery (SV/BV) & $28.2 / 6.11$ & & & \\
\hline$\%$ on medication $(\mathrm{SV} / \mathrm{BV})$ & $91 / 0$ & - & - & - \\
\hline
\end{tabular}

Note. $\mathrm{CHD}=$ congenital heart disease; $\mathrm{SV}=$ single ventricle; $\mathrm{BV}=$ viventricular; $d=$ Cohen's $d$ when applicable.

Cohen's d $=$ M1 $-\mathrm{M} 2 / \sigma_{\text {pooled }}$ where $\sigma_{\text {pooled }}=\sqrt{ }[(\sigma 12+\sigma 22) / 2] ; 0.2=$ small, $0.5=$ medium, $0.8=$ large $($ Cohen, 1988$)$. 
structural images (3D MPRAGE) in the sagittal plane, acquired with a three-dimensional magnetization-prepared rapid gradient echo sequence using the following parameters: repetition time/ echo time $=2250 / 3.98 \mathrm{~ms}, 176$ contiguous sagittal slices, $1 \mathrm{~mm}$ slice thickness, no gap, voxel size: $1 \mathrm{~mm}^{3}$.

\section{Image Processing}

Total intracranial volume (ICV) was determined with voxelbased morphometry in SPM8 and was considered as a covariate to account for individual difference in cranial size (Sanfilipo, Benedict, Zivadinov, \& Bakshi, 2004). We computed putamen volumes as a comparison region using this same method. Cerebellar volumes were analyzed using the SUIT toolbox for SPM, which was developed specifically to deal with high inter-subject anatomical variability in the CB (Diedrichsen, 2006). Preprocessing was done according to SUIT documentation, followed by isolation, normalization, and segmentation in the SUIT macro. Images were reviewed for quality and no aberrant $\mathrm{CB}$ morphology or lesions were noted. SUIT provided total $\mathrm{CB}$ volumes as well as the volumes of individual lobules using a probabilistic atlas.

\section{Neuropsychological Assessment}

EF was assessed with the Verbal Fluency, Trail Making, and Color-Word Interference (CWI) subscales from the Delis-Kaplan Executive Function System (D-KEFS) and the informant-report version of the Behavior Rating Inventory of Executive Function (BRIEF) Global Executive Composite (Delis, Kaplan, \& Kramer, 2001; Gioia, Isquith, Guy, \& Kenworthy, 2000). The Matrix Reasoning subscale from the Wechsler Abbreviated Scale of Intelligence, Second Edition (WASI-II) was used as an untimed measure of problem solving, abstract reasoning, and cognitive flexibility (Wechsler, 2011). While not traditionally viewed as a measure of EF, the Matrix Reasoning task has been cited as a valid assessment of EF because of its high reliance on these abilities (Schoenberg \& Scott, 2011). The Vocabulary subtest was analyzed to evaluate whether EF differences could be attributed to a general low level of cognitive functioning or whether EF appears to be differentially affected. The oral form of the Symbol Digit Modalities Test (SDMT) was used to assess processing speed in a way that was not susceptible to common motor deficits in CHD (Smith, 1982). Finally, dominant hand motor function was assessed using the Grooved Pegboard task (Lafayette Instrument Company Inc., 2002).

\section{Statistical Analyses}

Intracranial vault volume and several non-EF-related subtests from the D-KEFS were considered as confounds. We defined a confound as a variable that was significantly correlated with both the independent variable (CB volume) and the dependent variable (e.g., the EF score). IQ was not considered as a potential confound, following concerns raised by Dennis et al. (2009). Category Fluency and Visual Scanning significantly correlated with both Processing Speed and at least one dependent variable, therefore, they were controlled for in the respective regressions.

To test group differences one-tailed independent samples $t$ tests and Cohen's $d$ effect sizes were calculated, as we hypothesized a priori that $\mathrm{CHD}$ patients would have smaller $\mathrm{CB}$ volumes and lower performance. Proportions of individuals in the impaired range ( 1.5 or more standard deviations below the normative mean) were calculated and chi-square tests tested for significant differences in the proportion of impairment between the CHD and control groups. Pearson's correlations tested relationships between $\mathrm{CB}$ volume and neuropsychological outcomes and Fisher's $Z$ Transformations tested whether the correlations between the posterior $\mathrm{CB}$ and $\mathrm{EF}$ were significantly greater than with motor function and whether the correlation between anterior $\mathrm{CB}$ volume and motor function was significantly larger than those with EF.

These correlations were conducted across both groups and also within groups individually to determine whether the same pattern emerged for CHD and controls when analyzed separately. Hierarchical regression was used to test whether posterior $\mathrm{CB}$ volume explains a significant amount of variance in EF above and beyond processing speed. Finally, as an a priori exploratory analysis, analysis of variance assessed differences in our outcome variables between three levels of severity and planned Fisher's least significant difference tests were conducted for results with significant omnibus effects.

We also have included an examination of putamen volumes and associations with EF. We also sought to know whether the posterior $\mathrm{CB}$ predicts $\mathrm{EF}$ above and beyond putamen volumes. The putamen has been shown to project to the prefrontal cortex and to be involved in EF in previous research (Alexander, DeLong, \& Strick, 1986; Middleton \& Strick, 1994). It has also been shown that the basal ganglia communicate reciprocally with the CB (Bostan, Dum, \& Strick, 2010; Caligiore et al., 2017; Hoshi, Tremblay, Feger, Carras, \& Strick, 2005). Since there is no part of the basal ganglia that we would expect to be unrelated to EF, this serves as more of an informal comparison in contrast to our main control region, the anterior $\mathrm{CB}$.

\section{RESULTS}

There were no significant differences in demographic variables, Vocabulary subtest scores, or ICV (see Table 2). Individuals with $\mathrm{CHD}$ had smaller total, posterior, and anterior $\mathrm{CB}$ volumes than controls and reduced white matter. Although the effect size was moderate, there was no statistically significant difference in putamen volume. CHD patients performed significantly more poorly on three measures of EF: D-KEFS CWI Inhibition, Matrix Reasoning, and the BRIEF. Motor function was also significantly lower for individuals with CHD. A nonsignificant trend with a medium effect size suggested slower oral processing speed. On average, neither group was impaired on any of the neuropsychological measures; however, there were significantly more CHD participants who were impaired on the BRIEF and Grooved Pegboard. Control 
Table 2. CHD vs. controls: demographics, brain volumes, and neuropsychological performance

\begin{tabular}{|c|c|c|c|c|c|}
\hline & Variable & CHD & Control & $t / \chi^{2}$ & $d$ \\
\hline ICV & Total ICV (voxel units) & $1,931.2(84.48)$ & $1,937.8(207.7)$ & 0.14 & 0.04 \\
\hline \multirow[t]{5}{*}{$\mathrm{CB}$ vol. $\left(\mathrm{mm}^{3}\right)$} & Total CB volume & $64,465(14,293)$ & $77,381(18,853)$ & $2.56^{* *}$ & 0.78 \\
\hline & Posterior CB volume & $53,095(13,748)$ & $63,349(18,087)$ & $2.12 *$ & 0.64 \\
\hline & Anterior CB volume & $11,370(5,040)$ & $14,033(5,283)$ & $1.71 *$ & 0.52 \\
\hline & $\mathrm{CB}$ white matter volume & $18,146(5,039)$ & $23,325(5,409)$ & $3.29 * *$ & 0.99 \\
\hline & $\mathrm{CB}$ gray matter volume & $46,319(12,429)$ & $54,056(17,806)$ & 1.67 & 0.50 \\
\hline Putamen vol. $\left(\mathrm{mm}^{3}\right)$ & Total putamen volume & $10,278(1,369)$ & $10,937(1,320)$ & 1.62 & 0.49 \\
\hline \multirow[t]{8}{*}{ D-KEFS } & Category Switching & $0.07(1.16)$ & $0.30(0.96)$ & 0.71 & 0.22 \\
\hline & $\%$ Impaired & 13.6 & 4.5 & 1.10 & \\
\hline & Letter-Number Sequencing & $-0.39(1.28)$ & $0.03(0.90)$ & 1.27 & 0.39 \\
\hline & $\%$ Impaired & 18.2 & 4.5 & 2.03 & \\
\hline & CWI Inhibition & $-0.17(0.90)$ & $0.44(0.64)$ & $2.59 * *$ & 0.79 \\
\hline & $\%$ Impaired & 4.5 & 0.0 & 1.02 & \\
\hline & CWI Inhibition-Switching & $-0.17(0.88)$ & $0.23(0.93)$ & 1.44 & 0.44 \\
\hline & $\%$ Impaired & 9.1 & 4.5 & 0.36 & \\
\hline \multirow[t]{4}{*}{ WASI } & Matrix Reasoning & $-0.21(1.13)$ & $0.55(0.49)$ & $2.89 * *$ & 0.94 \\
\hline & $\%$ Impaired & 13.6 & 0.0 & 3.22 & \\
\hline & Vocabulary & $0.45(1.03)$ & $0.80(0.53)$ & 1.42 & 0.45 \\
\hline & $\%$ Impaired & 0.0 & 0.0 & - & \\
\hline \multirow[t]{2}{*}{ BRIEF } & General Executive Composite & $0.72(1.34)$ & $-0.37(0.84)$ & $-3.15 * *$ & 0.97 \\
\hline & $\%$ Impaired & 31.8 & 0.0 & $8.32 * *$ & \\
\hline \multirow[t]{2}{*}{ SDMT } & Oral scale & $0.89(2.04)$ & $0.01(1.42)$ & -1.65 & 0.51 \\
\hline & $\%$ Impaired & 4.5 & 9.1 & 0.31 & \\
\hline \multirow[t]{2}{*}{ Grooved Pegboard } & Dominant Hand & $-1.06(1.66)$ & $0.70(0.15)$ & $2.49 * *$ & 0.81 \\
\hline & $\%$ Impaired & 27.3 & 0.0 & $6.95 * *$ & \\
\hline
\end{tabular}

Note. $\mathrm{CHD}=$ congenital heart disease; $\mathrm{ICV}=$ intracranial vault volume; $\mathrm{CB}=$ cerebellum; vol. = volume; D-KEFS = Delis-Kaplan Executive Function System; WASI $=$ Wechsler Abbreviated Scale of Intelligence; BRIEF = Behavior Rating Inventory of Executive Function; SDMT = Symbol Digit Modalities Test; vol. $=$ volume.

Higher scores indicate better performance for all tests except the BRIEF and SDMT.

Neuropsychological test results reported as $Z$ scores. Cohen's d $=\mathrm{M} 1-\mathrm{M} 2 / \sigma_{\text {pooled }}$ where $\sigma_{\text {pooled }}=\sqrt{ }[(\sigma 12+\sigma 22) / 2] ; 0.2=$ small, $0.5=$ medium, $0.8=$ large (Cohen, 1988).

$* p<.05$.

$* * p<.01$

impairment reflected natural variability as opposed to one specific individual with broad EF impairment (Table 2).

For our correlation analyses, a similar pattern of findings emerged when analyzed across and within groups, therefore, we report across group results here. There were significant correlations between the following EF measures and the posterior CB only: Letter-Number Sequencing, CWI Inhibition, CWI Inhibition-Switching, and the BRIEF. Matrix Reasoning subscale was correlated with both the posterior and anterior $\mathrm{CB}$. The only measure of EF to have no relationship with $\mathrm{CB}$ volumes was Category Switching. Finally, the Grooved Pegboard was correlated only with the posterior CB. There were two significant Fisher's $Z$ Transformation results, indicating that both CWI Inhibition and CWI Inhibition-Switching were significantly more strongly associated with the posterior than the anterior CB. As a comparison, the putamen was significantly associated with three measures of EF: Color-Word Inhibition, Matrix Reasoning, and the BRIEF (Table 3).

Regression analyses examined whether posterior $\mathrm{CB}$ volumes predicted EF above and beyond processing speed alone. Two significant results emerged: CWI Inhibition $\left(\Delta \mathrm{R}^{2}=.240 ; p<.01\right)$ and Matrix Reasoning $\left(\Delta \mathrm{R}^{2}=.233\right.$; $p<.01)$. Analyses of whether the CB predicts EF above and beyond the putamen yielded three significant results, such that the $\mathrm{CB}$ predicted Letter-Number Sequencing $\left(\Delta \mathrm{R}^{2}=.091 ; p<.05\right)$, CWI Inhibition $\left(\Delta \mathrm{R}^{2}=.150 ; p<.01\right)$, and Matrix Reasoning $\left(\Delta \mathrm{R}^{2}=.144 ; p<.01\right)$ above and beyond the putamen and processing speed combined.

A priori exploratory analyses by CHD severity revealed four significant omnibus effects: putamen volume, CWI Inhibition, Matrix Reasoning, and the BRIEF. Additionally, there were medium-to-large effect sizes for differences in anterior and posterior CB volumes and Grooved Pegboard. For putamen volume, there were differences between controls and single ventricle (most severe) as well as between single ventricle and biventricular. For the three cognitive measures, there were significant differences only between controls and single ventricle (Table 4).

\section{DISCUSSION}

All measurements of $\mathrm{CB}$ volumes were significantly smaller in CHD patients as compared to controls, except total gray matter (which was a moderate effect). These findings confirm that this population is at risk for abnormal CB development. Additionally, no difference in ICV suggests that reductions in the $\mathrm{CB}$ are 
Table 3. Cerebellar lobe and putamen volume with executive and motor measure correlations

\begin{tabular}{|c|c|c|c|c|}
\hline & \multirow{2}{*}{$\frac{\text { Comparison region }}{\text { Total putamen vol. }}$} & \multicolumn{3}{|c|}{ Cerebellar regions of interest } \\
\hline & & Posterior CB vol. & Anterior CB vol. & Fisher's Z test (post vs. ant CB) \\
\hline D-KEFS Category Switching & -0.121 & -0.034 & 0.089 & 0.2877 \\
\hline D-KEFS Letter-Number Sequencing & 0.066 & $0.286^{*}$ & 0.15 & 0.2578 \\
\hline D-KEFS CWI Inhibition & $0.301 *$ & $0.483 * *$ & 0.063 & $0.0179 *$ \\
\hline D-KEFS CWI Inhibition-Switching & 0.216 & $0.292 *$ & -0.067 & $0.0475^{*}$ \\
\hline WASI-Matrix Reasoning & $0.384 *$ & $0.557 * *$ & $0.307 *$ & 0.0793 \\
\hline BRIEF General Executive Composite & $-0.351 *$ & $-0.253^{*}$ & -0.105 & 0.2451 \\
\hline Grooved Pegboard & 0.217 & $0.348^{*}$ & 0.184 & 0.2119 \\
\hline
\end{tabular}

Note. D-KEFS = Delis-Kaplan Executive Function System; CWI=Color-Word Interference; WASI = Wechsler Abbreviated Scale of Intelligence; CB = cerebellar; vol. $=$ volume; post $=$ posterior $;$ ant $=$ anterior .

Higher scores indicate better performance for all tests except the BRIEF

$* p<.05$.

$* * p<.01$

not the result of reduced total brain volume. The putamen showed a moderate effect size for reduction in volume, but this was not statistically significant. Measures of inhibition, abstract reasoning, and informant-reported EF were significantly lower for the CHD group. This supports previous research which has suggested that EF impairments persist into adolescence and young adulthood and confirms the importance of continued screening and follow-up.

Additionally, there was no significant group difference on Vocabulary, which supports the notion that CHD patients are differentially susceptible to EF deficits. Finally, CHD patients demonstrated reduced motor function, a finding that has been frequently documented. Overall, the highest rates of impairment for CHD were in informant-reported EF $(31.8 \%$ vs. $0 \%$ in controls) and motor function $(27.3 \%$ vs. $0 \%)$. On cognitive performance measures, the highest rates of impairment in CHD relative to controls were on a measure of cognitive flexibility ( $18.2 \% v s .4 .5 \%)$, followed by a measure of abstract reasoning ( $13.6 \%$ vs. $0 \%)$, however, these were not statistically significant and on average both groups performed within normal limits.

A consistent pattern emerged whereby EF measures of inhibition, cognitive flexibility, abstract reasoning, and informant-reported $\mathrm{EF}$ were correlated with the posterior $\mathrm{CB}$ but not the anterior. This constitutes robust evidence that there is a specific role for the posterior CB in EF. The only measure of EF that was associated with the anterior $\mathrm{CB}$ as well was Matrix Reasoning, a measure of problem solving and abstract reasoning that relies heavily on EF. This could be because this test also requires many non-EF abilities, including classification and spatial ability, knowledge of part-whole relationships, and perceptual organization (Wechsler, 2011). The putamen was correlated with three measures of EF, suggesting a slightly less robust (but still existent) relationship with $\mathrm{EF}$.

Motor function and its relationship to the $\mathrm{CB}$ did not confirm that these functions are related to the anterior $\mathrm{CB}$. This could be because this task requires elements of planning

Table 4. Diagnosis severity: analysis of variance omnibus effects

\begin{tabular}{|c|c|c|c|c|c|c|}
\hline & $\begin{array}{l}\text { Controls } \\
(N=22)\end{array}$ & $\begin{array}{l}\text { Biventricular } \\
\quad(N=12)\end{array}$ & $\begin{array}{l}\text { Single ventricle } \\
\qquad(N=10)\end{array}$ & $\mathrm{F}$ & $\eta^{2}$ & $p$-Value \\
\hline Posterior CB vol. $\left(\mathrm{mm}^{3}\right)$ & $63,348(18,087)$ & $57,251(8,826)$ & $48,107(17,180)$ & 3.18 & 0.13 & .052 \\
\hline Anterior CB vol. $\left(\mathrm{mm}^{3}\right)$ & $14,032(5,283)$ & $9,911(4,525)$ & $13,120(5,292)$ & 2.59 & 0.11 & .087 \\
\hline Total Putamen vol. $\left(\mathrm{mm}^{3}\right)$ & $10,936(1,320)$ & $10,946(1,176)$ & $9,477(1,174)$ & 5.28 & 0.21 & $.009 * *$ \\
\hline D-KEFS Category Switching & $0.30(0.96)$ & $-0.22(1.05)$ & $0.43(1.24)$ & 1.33 & 0.07 & .277 \\
\hline D-KEFS Letter-Number Sequencing & $0.03(0.90)$ & $-0.25(1.37)$ & $-0.57(1.20)$ & 1.02 & 0.05 & .369 \\
\hline D-KEFS CWI Inhibition & $0.44(0.64)$ & $0.00(0.57)$ & $-0.37(1.18)$ & 3.97 & 0.16 & $.026^{*}$ \\
\hline D-KEFS CWI Inhibition-Switching & $0.23(0.93)$ & $-0.14(0.48)$ & $-0.20(1.24)$ & 1.03 & 0.05 & .366 \\
\hline WASI Matrix Reasoning & $0.55(0.49)$ & $-0.01(0.99)$ & $-0.45(1.28)$ & 4.94 & 0.19 & $.012^{*}$ \\
\hline BRIEF GEC & $-0.38(0.84)$ & $0.33(1.26)$ & $1.20(1.33)$ & 7.00 & 0.26 & $.003 * *$ \\
\hline Oral Systems Digit Modalities & $0.01(1.42)$ & $1.17(2.04)$ & $0.58(2.11)$ & 1.64 & 0.08 & .206 \\
\hline Grooved Pegboard & $-0.10(0.70)$ & $-1.15(1.83)$ & $-0.96(1.51)$ & 3.10 & 0.13 & .056 \\
\hline
\end{tabular}

Note . $\mathrm{CB}=$ cerebellar; vol. = volume, D-KEFS $=$ Delis-Kaplan Executive Function System; $\mathrm{CWI}=$ Color-Word Interference; WASI $=$ Wechsler Abbreviated Scale of Intelligence; BRIEF GEC = Behavior Rating Inventory of Executive Function General Executive Composite.

Neuropsychological test results reported as $Z$ scores.

$* p<.05$.

$* * p<.01$. 
and visual processing that might be related to posterior $\mathrm{CB}$ function. It is possible that Finger Tapping or a similar, more automatic motor task may have better dissociated motor function. Regardless, this does not explain the lack of an association with the anterior CB. Out of curiosity, a post hoc correlation between the anterior $\mathrm{CB}$ and the Motor Speed subtest of the D-KEFS was conducted as an alternative, which was nonsignificant. Therefore, it could be that there is no relationship between motor function and the anterior $\mathrm{CB}$ (which would contradict Stoodley and Schmahmann's model of $\mathrm{CB}$ function), we may have had insufficient power, or there could be motor disruption in another part of the brain. It is also possible that neuroimaging methods other than volumetrics would contribute different valuable information.

Fisher's Z Transformations showed that both CWI subtests were significantly more highly correlated with the posterior $\mathrm{CB}$ than with the anterior $\mathrm{CB}$. This suggests that these two tests have the strongest specificity to the posterior $\mathrm{CB}$ out of all the EF assessments. What these tests have most in common is that they draw heavily on the ability to inhibit a prepotent response in favor of a correct response. Therefore, it appears likely that the posterior CB may be participating in inhibitory mechanisms. Despite no other significant findings, there were other large and meaningful differences between posterior and anterior correlations, suggesting additional research is warranted.

Past studies of the CB's involvement in cognitive efficiency have used processing speed as a proxy indicator of this construct; however, there are also task-specific abilities that are necessary to exhibit cognitive efficiency. Therefore, we wanted to explore whether the $\mathrm{CB}$ contributes any of these task-specific elements above and beyond processing speed. When added to a model with processing speed, the posterior $\mathrm{CB}$ explained a significant amount of additional variance in inhibitory abilities and abstract reasoning, supporting our hypothesis that the posterior CB contributes EF-specific functions in addition to facilitating speed.

Particularly notable was that the regression predicting CWI Inhibition was significant, as this lends further support to the proposition that the posterior $\mathrm{CB}$ contributes to inhibition. A potential mechanism for this involvement in response inhibition was proposed by Bellebaum and Daum (2007), who noted the strong reciprocal connections between the $\mathrm{CB}$ and the lateral prefrontal cortex which, when lesioned, often results in reduced response inhibition. Several studies have found that individuals with a variety of frontal lesions were impaired on measures of inhibition and that this effect is strongest for lateral prefrontal lesions (Aron, Fletcher, Bullmore, Sahakian, \& Robbins, 2003; Burgess \& Shallice, 1996; Vendrell et al., 1995). Studies of inhibition in individuals with CB lesions have been less consistent (Heyder, Suchan, \& Daum, 2004; Neau, Arroyo-Anllo, Bonnaud, Ingrand, \& Gil, 2000). This points to a need for further work to understand the CB's role in inhibition.

Concerning the significant regression predicting performance on Matrix Reasoning, it is possible that participants with smaller posterior CB volumes had compromised spatial problem solving and abstract reasoning. These EF skills are essential to successful Matrix Reasoning performance and have been noted as commonly impaired in individuals with $\mathrm{CB}$ lesions (Petrosini, Leggio, \& Molinari, 1998). Kalbfleisch, Van Meter, and Zeffiro (2007) found BOLD responses in lobule VI of the $\mathrm{CB}$ (part of the posterior lobe) during a Matrix Reasoning task. They proposed that the $\mathrm{CB}$ is important in reasoning in the absence of explicit external feedback, and that under conditions of uncertainty, the CB forms internal models of response correctness, serving as a guide to the brain during complex and novel problem solving. They note that this is particularly true under time-pressured conditions.

Our findings that the posterior CB predicted three of our EF assessments above and beyond the putamen in addition to processing speed was an unexpected finding. This suggests that the $\mathrm{CB}$ accounts for more variation in some aspects of $\mathrm{EF}$ than the putamen. One potential explanation for this is that the CB projects directly to the striatum by way of the thalamus, potentially modulating its function and its output to the prefrontal cortex. The putamen's influence over the $\mathrm{CB}$ is more indirect, as it does not project directly to the $\mathrm{CB}$ (Caligiore et al., 2017). The interaction of these structures with respect to EF should be the focus of future study.

Several outcomes seem to be impacted by the severity of the CHD diagnosis, including $\mathrm{CB}$ and putamen volumes, inhibitory and global EF abilities, and motor function. For the EF measures, only controls and single ventricle patients were significantly different from one another. This finding is consistent with previous research that has found that more severe diagnoses result in more profound impairment (Karsdorp, Everaerd, Kindt, \& Mulder, 2007). Therefore, it would appear that a subset of $\mathrm{CHD}$ patients is at relatively low risk for cognitive impairment, while others are more likely to experience difficulty. While on average the most severe diagnosis group was not impaired on any measures, it did tend to have the greatest number of impaired individuals. That being said, more research is needed given the potentially subtle differences in EF outcomes in less severe CHD diagnoses.

The current study's findings should be considered within the context of its limitations. First, these data are cross-sectional, making it difficult to draw any firm conclusions about causality or the direction of effects other than those based in theory. Our sample is modest, although this is in fact a respectable sample size in the context of neuroimaging studies of medical populations. We conducted a post hoc power analysis to provide more context for negative findings. We found that, while our sample size of 44 is a statistical limitation, it should have been able to detect correlations as low as 0.35 .

Many strengths of the current study should be highlighted to emphasize its contribution to the field. First, we excluded individuals who were born prematurely, which is not always a consideration in studies of CHD. Prematurity poses a serious confound when evaluating neuroimaging results, as it confers risk for intraventricular hemorrhage. We also excluded individuals with developmental disorders (many of which are due to genetic disorders that also cause CHD). These exclusions allowed us to more confidently make conclusions about CHD-specific impairment. Another strength is 
that our hypotheses are theory-driven and firmly based in the current literature. The study also extended knowledge on the involvement of the $\mathrm{CB}$ in cognition. Additionally, we used image analysis that has been specifically developed to study the $\mathrm{CB}$, which maximizes accuracy. Finally, given that EF is a broad domain of cognitive functions, we examined multiple subtypes of EF (e.g., inhibition, cognitive flexibility, fluency) from both performance and informant-report methods.

\section{CONCLUSIONS AND FUTURE DIRECTIONS}

This study adds to the sparse literature on adolescent and young adult neurocognitive outcomes in CHD. As compared to healthy controls, these individuals are at risk for a variety of negative outcomes, including reduced $\mathrm{CB}$ volumes, impaired EF abilities, and poorer motor function. The findings of a single dissociation such that our EF measurements were related to the posterior $\mathrm{CB}$ but not the anterior $\mathrm{CB}$ suggest a role for the $\mathrm{CB}$ in higher order cognition. We were unable to confirm our hypothesized double dissociation between the posterior and anterior $\mathrm{CB}$, as the posterior $\mathrm{CB}$ was related to motor function and the anterior was not. Furthermore, we found that posterior CB volumes were able to predict $\mathrm{EF}$ above and beyond processing speed, suggesting that the $\mathrm{CB}$ contributes to $\mathrm{EF}$ beyond simply speeding processing.

The CB also predicted EF above and beyond putamen volumes and processing speed combined, suggesting a potentially unique role for the $\mathrm{CB}$ in some aspects of $\mathrm{EF}$ beyond other subcortical structures. Finally, patients with severe CHDs fare the worst and accounted for the majority of individuals falling into the impaired range, while more mild-moderate CHDs exhibited more subtle impairment. Clinicians assessing CHD patients should be mindful of subtle effects that nevertheless could have important influence on daily functioning and quality of life.

These findings help to lay the groundwork for more research. Future work should explore $\mathrm{CB}$ anatomy in $\mathrm{CHD}$ and relationships between the $\mathrm{CB}$ and cognitive outcomes using complementary techniques, such as fMRI and diffusion tensor imaging. Additionally, the mechanisms behind the CB's contribution to inhibitory processes and cognitive control remain unclear. Future work should also aim to identify more specific medical factors (e.g., number of surgeries) and their relationship to $\mathrm{CB}$ and EF outcomes. The identification of modifiable risk factors is essential to improving long-term outcomes. Finally, future work should continue to examine other subcortical structures, as our findings here suggest some involvement of the putamen, although the unique roles of the various subcortical structures is unclear.

\section{ACKNOWLEDGMENTS}

We thank the students in Dr. King's Developmental Neuropsychology Across the Lifespan (DNP-ATL) Laboratory who assisted with acquiring data for this study. This research was supported by the Children's Healthcare of Atlanta Cardiovascular
Biology Research Center Seed Grant (W.M., PI), the American Cancer Society (Research Scholar Grant \#114044-RSGPB-07-17001-CPPB; T.K., PI), and the GSU Brains \& Behavior graduate student fellowship (E.S.). The authors declare no conflicts of interest.

\section{REFERENCES}

Ailion, A.S., King, T.Z., Wang, L., Fox, M.E., Mao, H., Morris, R.M., \& Crosson, B. (2016). Cerebellar atrophy in adult survivors of childhood cerebellar tumor. Journal of the International Neuropsychological Society, 22(5), 501-511. doi: 10.1017/S13556 17716000138

Alexander, G.E., DeLong, M.R., \& Strick, P.L. (1986). Parallel organization of functionally segregated circuits linking basal ganglia and cortex. Annual Review of Neuroscience, 9, 357-381. doi: 10.1146/annurev.ne.09.030186.002041

Allin, M., Matsumoto, H., Santhouse, A.M., Nosarti, C., AlAsady, M.H.S., Stewart, A.L., ... Murray, R.M. (2001). Cognitive and motor function and the size of the cerebellum in adolescents born very pre-term. Brain, 124(1), 60-66. doi: 10.1093/brain/124.1.60

Aron, A.R., Fletcher, P.C., Bullmore, E.T., Sahakian, B.J., \& Robbins, T.W. (2003). Stop-signal inhibition disrupted by damage to right inferior frontal gyrus in humans. Nature Neuroscience, 6(2), 115-116. doi: 10.1038/nn1003

Barker, P.C., Nowak, C., King, K., Mosca, R.S., Bove, E.L., \& Goldberg, C.S. (2005). Risk factors for cerebrovascular events following fontan palliation in patients with a functional single ventricle. American Journal of Cardiology, 96(4), 587-591. doi: 10.1016/j.amjcard.2005.04.025

Beca, J., Gunn, J.K., Coleman, L., Hope, A., Reed, P.W., Hunt, R.W., ... Shekerdemian, L.S. (2013). New white matter brain injury after infant heart surgery is associated with diagnostic group and the use of circulatory arrest. Circulation, 127(9), 971-979. doi: 10.1161/ CIRCULATIONAHA.112.001089

Bellebaum, C., \& Daum, I. (2007). Cerebellar involvement in executive control. Cerebellum, 6(3), 184-192. doi: 10.1080/ 14734220601169707

Bellinger, D.C., Wypij, D., Rivkin, M.J., DeMaso, D.R., Robertson, R.L. Jr., Dunbar-Masterson, C., .. Newburger, J.W. (2011). Adolescents with d-transposition of the great arteries corrected with the arterial switch procedure: Neuropsychological assessment and structural brain imaging. Circulation, 124(12), 1361-1369. doi: 10.1161/CIRCULATIONAHA.111.026963

Bergemann, A., Hansen, J.H., Rotermann, I., Voges, I., Scheewe, J., Otto-Morris, C., ... Kramer, H.H. (2015). Neuropsychological performance of school-aged children after staged surgical palliation of hypoplastic left heart syndrome. European Journal of Cardio-thoracic Surgery, 47(5), 803-811. doi: 10.1093/ejcts/ ezu299

Bolduc, M.E., du Plessis, A.J., Sullivan, N., Guizard, N., Zhang, X., Robertson, R.L., \& Limperopoulos, C. (2012). Regional cerebellar volumes predict functional outcome in children with cerebellar malformations. Cerebellum, 11(2), 531-542. doi: 10.1007/s12311-011-0312-z

Bostan, A.C., Dum, R.P., \& Strick, P.L. (2010). The basal ganglia communicate with the cerebellum. Proceedings of the National Academy of Sciences of the United States of America, 107(18), 8452-8456. doi: 10.1073/pnas.1000496107

Brewster, R.C., King, T.Z., Burns, T.G., Drossner, D.M., \& Mahle, W.T. (2015). White matter integrity dissociates verbal memory and auditory attention span in emerging adults with congenital 
heart disease. Journal of the International Neuropsychological Society, 21(1), 22-33. doi: 10.1017/S135561771400109X

Burgess, P.W., \& Shallice, T. (1996). Response suppression, initiation and strategy use following frontal lobe lesions. Neuropsychologia, 34(4), 263-272. doi:Doi 10.1016/0028-3932 (95)00104-2

Caligiore, D., Pezzulo, G., Baldassarre, G., Bostan, A.C., Strick, P.L., Doya, K., ... Herreros, I. (2017). Consensus paper: Towards a systems-level view of cerebellar function: The interplay between cerebellum, basal ganglia, and cortex. Cerebellum, 16(1), 203-229. doi: 10.1007/s12311-016-0763-3

Cassidy, A.R., White, M.T., DeMaso, D.R., Newburger, J.W., \& Bellinger, D.C. (2015). Executive function in children and adolescents with critical cyanotic congenital heart disease. Journal of the International Neuropsychological Society, 21(1), 34-49. doi: 10.1017/S1355617714001027

Cohen, J. (1988). Statistical power analysis for the behavioral science (2nd ed.). Hillsdale, NJ: Lawrence Earlbaum Associates.

Daliento, L., Mapelli, D., Russo, G., Scarso, P., Limongi, F., Iannizzi, P., ... Volpe, B. (2005). Health related quality of life in adults with repaired tetralogy of Fallot: Psychosocial and cognitive outcomes. Heart, 91(2), 213-218. doi: 10.1136/hrt.2003.029280

Delis, D.C., Kaplan, E., \& Kramer, J.H. (2001). Delis-Kaplan Executive Function System (D-KEFS). San Antonio, TX: Pearson.

Dennis, M., \& Barnes, M.A. (2010). The cognitive phenotype of spina bifida meningomyelocele. Developmental Disabilities Research Reviews, 16(1), 31-39. doi: 10.1002/ddrr.89

Dennis, M., Francis, D.J., Cirino, P.T., Schachar, R., Barnes, M.A., \& Fletcher, J.M. (2009). Why IQ is not a covariate in cognitive studies of neurodevelopmental disorders. Journal of the International Neuropsychological Society, 15(3), 331-343. doi: 10.1017/ S1355617709090481

Diedrichsen, J. (2006). A spatially unbiased atlas template of the human cerebellum. Neuroimage, 33(1), 127-138. doi: 10.1016/j. neuroimage.2006.05.056

du Plessis, A.J. (1999). Mechanisms of brain injury during infant cardiac surgery. Seminars in Pediatric Neurology, 6(1), 32-47. doi: 10.1016/s1071-9091(99)80045-x

Gaynor, J.W., Gerdes, M., Nord, A.S., Bernbaum, J., Zackai, E., Wernovsky, G., ... Jarvik, G.P. (2010). Is cardiac diagnosis a predictor of neurodevelopmental outcome after cardiac surgery in infancy? The Journal of Thoracic and Cardiovascular Surgery, 140(6), 1230-1237. doi: 10.1016/j.jtcvs.2010.07.069

Geyer, S., Norozi, K., Buchhorn, R., \& Wessel, A. (2009). Chances of employment in women and men after surgery of congenital heart disease: Comparisons between patients and the general population. Congenital Heart Disease, 4(1), 25-33. doi: 10.1111/ j.1747-0803.2008.00239.x

Gilboa, S.M., Salemi, J.L., Nembhard, W.N., Fixler, D.E., \& Correa, A. (2010). Mortality resulting from congenital heart disease among children and adults in the United States, 1999 to 2006. Circulation, 122(22), 2254-2263. doi: 10.1161/ CIRCULATIONAHA.110.947002

Gioia, G.A., Isquith, P.K., Guy, S.C., \& Kenworthy, L. (2000). Behavior rating inventory of executive function. Odessa, FL: Psychological Assessment Resources.

Heyder, K., Suchan, B., \& Daum, I. (2004). Cortico-subcortical contributions to executive control. Acta Psychologica, 115(2-3), 271-289. doi: 10.1016/j.actpsy.2003.12.010

Hoffman, J.I., Kaplan, S., \& Liberthson, R.R. (2004). Prevalence of congenital heart disease. American Heart Journal, 147(3), 425-439. doi: 10.1016/j.ahj.2003.05.003
Hoshi, E., Tremblay, L., Feger, J., Carras, P.L., \& Strick, P.L. (2005). The cerebellum communicates with the basal ganglia. Nature Neuroscience, 8(11), 1491-1493. doi: 10.1038/nn1544

Ilardi, D., Ono, K.E., McCartney, R., Book, W., \& Stringer, A.Y. (2017). Neurocognitive functioning in adults with congenital heart disease. Congenital Heart Disease, 12(2), 166-173. doi: 10.1111/chd.12434

Jackson, J.L., Misiti, B., Bridge, J.A., Daniels, C.J., \& Vannatta, K. (2015). Emotional functioning of adolescents and adults with congenital heart disease: A meta-analysis. Congenital Heart Disease, 10(1), 2-12. doi: 10.1111/chd.12178

Juranek, J., Dennis, M., Cirino, P.T., El-Messidi, L., \& Fletcher, J.M. (2010). The cerebellum in children with spina bifida and Chiari II malformation: Quantitative volumetrics by region. Cerebellum, 9 (2), 240-248. doi: 10.1007/s12311-010-0157-x

Kalbfleisch, M.L., Van Meter, J.W., \& Zeffiro, T.A. (2007). The influences of task difficulty and response correctness on neural systems supporting fluid reasoning. Cognitive Neurodynamics, 1(1), 71-84. doi: 10.1007/s11571-006-9007-4

Karsdorp, P.A., Everaerd, W., Kindt, M., \& Mulder, B.J. (2007). Psychological and cognitive functioning in children and adolescents with congenital heart disease: A meta-analysis. Journal of Pediatric Psychology, 32(5), 527-541. doi: 10.1093/jpepsy/ js1047

King, T.Z., Na, S., \& Mao, H. (2015). Neural underpinnings of working memory in adult survivors of childhood brain tumors. Journal of the International Neuropsychological Society, 21(7), 494-505. doi: 10.1017/S135561771500051X

King, T.Z., Smith, K.M., Burns, T.G., Sun, B., Shin, J., Jones, R.A., ... Mahle, W.T. (2016). fMRI investigation of working memory in adolescents with surgically treated congenital heart disease. Applied Neuropsychology. Child, 6(1), 7-21. doi: 10.1080/216 22965.2015.1065185

Klouda, L., Franklin, W.J., Saraf, A., Parekh, D.R., \& Schwartz, D.D. (2017). Neurocognitive and executive functioning in adult survivors of congenital heart disease. Congenital Heart Disease, 12(1), 91-98. doi: 10.1111/chd.12409

Koziol, L.F., Budding, D., Andreasen, N., D'Arrigo, S., Bulgheroni, S., Imamizu, H., ... Yamazaki, T. (2014). Consensus paper: The cerebellum's role in movement and cognition. Cerebellum, 13(1), 151-177. doi: 10.1007/s12311-013-0511-x

Koziol, L.F., Budding, D.E., \& Chidekel, D. (2010). Adaptation, expertise, and giftedness: Towards an understanding of cortical, subcortical, and cerebellar network contributions. Cerebellum, 9(4), 499-529. doi: 10.1007/s12311-010-0192-7

Koziol, L.F., Budding, D.E., \& Chidekel, D. (2012). From movement to thought: Executive function, embodied cognition, and the cerebellum. Cerebellum, 11(2), 505-525. doi: 10.1007/ s12311-011-0321-y

Licht, D.J., Wang, J., Silvestre, D.W., Nicolson, S.C., Montenegro, L.M., Wernovsky, G., ... Detre, J.A. (2004). Preoperative cerebral blood flow is diminished in neonates with severe congenital heart defects. The Journal of Thoracic and Cardiovascular Surgery, 128(6), 841-849. doi: 10.1016/j.jtcvs.2004.07.022

Limperopoulos, C., Bassan, H., Gauvreau, K., Robertson, R.L. Jr., Sullivan, N.R., Benson, C.B., ... duPlessis, A.J. (2007). Does cerebellar injury in premature infants contribute to the high prevalence of long-term cognitive, learning, and behavioral disability in survivors? Pediatrics, 120(3), 584-593. doi: 10.1542/ peds.2007-1041

Limperopoulos, C., Soul, J.S., Gauvreau, K., Huppi, P.S., Warfield, S.K., Bassan, H., ... du Plessis, A.J. (2005). Late gestation cerebellar 
growth is rapid and impeded by premature birth. Pediatrics, 115(3), 688-695. doi: 10.1542/peds.2004-1169

Mahle, W.T., Clancy, R.R., Moss, E.M., Gerdes, M., Jobes, D.R., \& Wernovsky, G. (2000). Neurodevelopmental outcome and lifestyle assessment in school-aged and adolescent children with hypoplastic left heart syndrome. Pediatrics, 105(5), 1082-1089. doi: 10.1542/peds.105.5.1082

Mahle, W.T., Tavani, F., Zimmerman, R.A., Nicolson, S.C., Galli, K.K., Gaynor, J.W., ... Kurth, C.D. (2002). An MRI study of neurological injury before and after congenital heart surgery. Circulation, 106(12 Suppl 1), I109-I114.

Mahone, E.M., Zabel, T.A., Levey, E., Verda, M., \& Kinsman, S. (2002). Parent and self-report ratings of executive function in adolescents with myelomeningocele and hydrocephalus. Child Neuropsychology, 8(4), 258-270. doi: 10.1076/chin.8.4.258. 13510

Marelli, A.J., Mackie, A.S., Ionescu-Ittu, R., Rahme, E., \& Pilote, L. (2007). Congenital heart disease in the general population: Changing prevalence and age distribution. Circulation, 115(2), 163-172. doi: 10.1161/CIRCULATIONAHA.106.627224

Martin, S., \& Kitzman, P. (2017). Evidence of cerebellar dysfunction in children with myelomeningocele. Physical Medicine and Rehabilitation - International, 4(1).

Mebius, M.J., Kooi, E.M.W., Bilardo, C.M., \& Bos, A.F. (2017). Brain injury and neurodevelopmental outcome in congenital heart disease: A systematic review. Pediatrics, 140(1) doi: 10.1542/ peds.2016-4055

Middleton, F., \& Strick, P. (1994). Anatomical evidence for cerebellar and basal ganglia involvement in higher cognitive function. Science, 266(5184), 458-461. doi: 10.1126/science. 7939688

Murdaugh, D.L., King, T.Z., \& O’Toole, K. (2017). The efficacy of a pilot pediatric cognitive remediation summer program to prepare for transition of care. Child Neuropsychology, 24, 1-21. doi: 10.1080/09297049.2017.1391949

Neau, J.P., Arroyo-Anllo, E., Bonnaud, V., Ingrand, P., \& Gil, R. (2000). Neuropsychological disturbances in cerebellar infarcts. Acta Neurologica Scandinavica, 102(6), 363-370.

Ortinau, C., Beca, J., Lambeth, J., Ferdman, B., Alexopoulos, D., Shimony, J.S., ... Inder, T. (2012). Regional alterations in cerebral growth exist preoperatively in infants with congenital heart disease. The Journal of Thoracic and Cardiovascular Surgery, 143(6), 1264-1270. doi: 10.1016/j.jtcvs.2011.10.039

Owen, M., Shevell, M., Donofrio, M., Majnemer, A., McCarter, R., Vezina, G., ... Limperopoulos, C. (2014). Brain volume and neurobehavior in newborns with complex congenital heart defects. Journal of Pediatrics, 164(5), 1121-1127 e1121. doi: 10.1016/j.jpeds.2013.11.033

Petrosini, L., Leggio, M.G., \& Molinari, M. (1998). The cerebellum in the spatial problem solving: A co-star or a guest star? Progress in Neurobiology, 56(2), 191-210.

Peyvandi, S., De Santiago, V., Chakkarapani, E., Chau, V., Campbell, A., Poskitt, K.J., ... McQuillen, P. (2016). Association of Prenatal Diagnosis of Critical Congenital Heart Disease With Postnatal Brain Development and the Risk of Brain Injury. JAMA Pediatrics, 170(4), e154450. doi: 10.1001/jama pediatrics.2015.4450

Sanfilipo, M.P., Benedict, R.H., Zivadinov, R., \& Bakshi, R. (2004). Correction for intracranial volume in analysis of whole brain atrophy in multiple sclerosis: The proportion vs. residual method. Neuroimage, 22(4), 1732-1743. doi: 10.1016/j.neuroimage. 2004.03.037
Sanz, J.H., Berl, M.M., Armour, A.C., Wang, J., Cheng, Y.I., \& Donofrio, M.T. (2017). Prevalence and pattern of executive dysfunction in school age children with congenital heart disease. Congenital Heart Disease, 12(2), 202-209. doi: 10.1111/chd.12427

Schall, U., Johnston, P., Lagopoulos, J., Jüptner, M., Jentzen, W., Thienel, R., ... Ward, P.B. (2003). Functional brain maps of Tower of London performance: A positron emission tomography and functional magnetic resonance imaging study. Neuroimage, 20(2), 1154-1161. doi: 10.1016/s1053-8119(03)00338-0

Schoenberg, M.R., \& Scott, J.G. (2011). The little black book of neuropsychology: A syndrome-based approach. New York, NY: Springer.

Smith, A. (1982). Symbol Digit Modalities Test. Los Angeles, CA: Western Psychological Services.

Stoodley, C.J., \& Schmahmann, J.D. (2010). Evidence for topographic organization in the cerebellum of motor control versus cognitive and affective processing. Cortex, 46(7), 831844. doi: 10.1016/j.cortex.2009.11.008

Sun, L., Macgowan, C.K., Sled, J.G., Yoo, S.J., Manlhiot, C., Porayette, P., ... Seed, M. (2015). Reduced fetal cerebral oxygen consumption is associated with smaller brain size in fetuses with congenital heart disease. Circulation, 131(15), 1313-1323. doi: 10.1161/CIRCULATIONAHA.114.013051

Tyagi, M., Austin, K., Stygall, J., Deanfield, J., Cullen, S., \& Newman, S.P. (2014). What do we know about cognitive functioning in adult congenital heart disease? Cardiology in the Young, 24(1), 13-19. doi: 10.1017/S1047951113000747

van Rijen, E.H., Utens, E.M., Roos-Hesselink, J.W., Meijboom, F.J., van Domburg, R.T., Roelandt, J.R., ... Verhulst, F.C. (2003). Psychosocial functioning of the adult with congenital heart disease: A 20-33 years follow-up. European Heart Journal, 24(7), 673-683.

Vendrell, P., Junque, C., Pujol, J., Jurado, M.A., Molet, J., \& Grafman, J. (1995). The role of prefrontal regions in the Stroop task. Neuropsychologia, 33(3), 341-352.

Volpe, J.J. (2009). Cerebellum of the premature infant: Rapidly developing, vulnerable, clinically important. Journal of Child Neurology, 24(9), 1085-1104. doi: 10.1177/0883073809338067

von Rhein, M., Kugler, J., Liamlahi, R., Knirsch, W., Latal, B., \& Kaufmann, L. (2014). Persistence of visuo-constructional and executive deficits in adolescents after open-heart surgery. Research in Developmental Disabilities, 36C, 303-310. doi: 10.1016/j.ridd.2014.10.027

Wechsler, D. (2011). Wechsler Abbreviated Scale of Intelligence Second Edition manual. Bloomington, MN: Pearson.

Williams, R.V., Ravishankar, C., Zak, V., Evans, F., Atz, A.M., Border, W.L. ... Pediatric Heart Network, I. (2010). Birth weight and prematurity in infants with single ventricle physiology: Pediatric heart network infant single ventricle trial screened population. Congenital Heart Disease, 5(2), 96-103. doi: 10.1111/j.1747-0803.2009.00369.x

Wray, J. (2001). Congenital heart disease and cardiac surgery in childhood: Effects on cognitive function and academic ability. Heart, 85(6), 687-691. doi: 10.1136/heart.85.6.687

Yang, Q., Chen, H., Correa, A., Devine, O., Mathews, T.J., \& Honein, M.A. (2006). Racial differences in infant mortality attributable to birth defects in the United States, 1989-2002. Birth Defects Research. Part A, Clinical and Molecular Teratology, 76(10), 706-713. doi: 10.1002/bdra.20308

Zeng, S., Zhou, Q.C., Zhou, J.W., Li, M., Long, C., \& Peng, Q.H. (2015). Volume of intracranial structures on three-dimensional ultrasound in fetuses with congenital heart disease. Ultrasound in Obstetrics \& Gynecology, 46(2), 174-181. doi: 10.1002/uog.14677 